\section{Aspectos da biologia reprodutiva de Boa constrictor constrictor: um estudo histológico dos testículos nos períodos reprodutivos de quiescência e máxima atividade}

[Boa constrictor constrictor reproductive biology aspects: the testes histological study in the reproductive periods of quiescence and maximal activity]

\author{
H.J. Bento ${ }^{1,3}$, A. Ferreira ${ }^{2}$, F.F. Curcio ${ }^{2}$, M. Mehanna ${ }^{2}$, G.A. Iglesias ${ }^{1,3}$, R.C.R. Paz \\ ${ }^{1}$ Programa de pós-graduação - Universidade Federal de Mato Grosso - Cuiabá, MT \\ ${ }^{2}$ Instituto de Biociências - Universidade Federal de Mato Grosso - Cuiabá, MT \\ ${ }^{3}$ Faculdade de Medicina Veterinária - Universidade Federal de Mato Grosso - Cuiabá, MT
}

Heitor José Bento http://orcid.org/0000-0002-8670-1515 Adelina Ferreira

https://orcid.org/0000-0002-7999-4946 Felipe Franco Curcio Mahmoud Mehana
https://orcid.org/0000-0003-3746-8590 Gabriella Accardi Iglesias http://orcid.org/0000-0001-6578-0456 Regina Celia Rodrigues da Paz http://orcid.org/0000-0003-4567-0043

\title{
RESUMO
}

As serpentes pertencem ao segundo maior grupo dentro dos répteis, podendo apresentar sazonalidade quanto à espermatogênese, com produção descontínua ou contínua. O presente trabalho tem como objetivo caracterizar aspectos da biologia reprodutiva de Boa constrictor constrictor com base nos achados histológicos dos testículos nos períodos de máxima atividade (período de gametogênese) e quiescência. Os testículos de dois espécimes de Boa c. constrictor (7767 e 11752) foram cortados a uma espessura de $3 \mu \mathrm{m}$ em micrótomo, corados com azul de toluidina $1 \%$, fotodocumentados e descritos. A presença de espermatozoides na luz do túbulo seminífero no indivíduo 7767 indica um período de máxima gametogênese, enquanto o lúmen dos túbulos seminíferos pouco evidentes, sem a presença de espermatozoides e de células gaméticas em divisão, caracteriza o indivíduo 11752 em período quiescente. Mediante os achados histológicos descritos no presente estudo, concluiu-se que Boa c. constrictor apresenta sazonalidade em relação à gametogênese, sendo esse padrão de sazonalidade associado ao período de cópulas relatado em literatura característico de serpentes com padrão pré-nupcial.

Palavras-chave: serpente, reprodução, espermatogênese, pré-nupcial

\begin{abstract}
These snakes belong to the second largest group within the reptiles, being able to present seasonality regarding spermatogenesis, with discontinuous or continuous production. The present study aims to characterize Boa constrictor constrictor reproductive biology aspects from histological findings in testicles during periods of maximum activity (period of gametogenesis) and quiescence. The testicles of two specimens of Boa c. constrictor (7767 and 11752) were cut to a thickness of $3 \mu \mathrm{m}$ in microtome, stained with $1 \%$ toluidine blue, photodocumented and described. The spermatozoa presence in the seminiferous tubule lumen in individual 7767 indicates a period of maximum gametogenesis, whereas the seminiferous tubules lumen is not very evident without spermatozoa and the absence of dividing gametic cells characterizes individual 11752 in the quiescent period. Through the histological findings we concluded that Boa c. constrictor presents seasonality in relation to gametogenesis, and the pattern of reproductive seasonality observed along with the period of copulas reported in the literature resembles the pre-nuptial pattern.
\end{abstract}

Keywords: snake, reproduction, spermatogenesis, pre-nuptial

\section{INTRODUÇÃO}

Com aproximadamente 3700 espécies, as serpentes, pertencentes à Ordem Squamata, representam o segundo grupo mais diverso dentro dos répteis (Uetz et al., 2018). No Brasil são encontradas 371 espécies de serpentes,

Recebido em 31 de agosto de 2018

Aceito em 17 de janeiro de 2019

E-mail: heitorjbento@gmail.com agrupadas em 81 gêneros diferentes pertencentes a 10 famílias, sendo a família Boidae de grande interesse na manutenção em cativeiro no Brasil, não só pelo caráter conservacionista, mas também comercial, visto que são animais não peçonhentos e atualmente comercializados como pets exóticos (Bérnils e Costa, 2012; Grego et al., 2014). 
Serpentes pertencentes à família Boidae possuem distribuição mundial, sendo encontrada nas Américas, Índia, África Central, sul da Ásia e ilhas do Pacífico Sul; entre as espécies pertencentes a essa família, a jiboia (Boa constrictor) é encontrada desde o México até o norte da Argentina, sendo amplamente distribuída no Brasil. Alimenta-se de pequenos mamíferos, aves e lagartos; caracteriza-se por hábitos diurnos e noturnos e por ter como habitat áreas terrícolas, arborícolas, de cerrado e caatinga (Zug et al., 2001; Grego et al., 2014).

Serpentes podem apresentar sazonalidade quanto à espermatogênese, com produção espermática contínua ou descontínua (Pizzatto et al., 2006). A caracterização histológica da gametogênese nesses animais permite avaliar os dados referentes aos índices gonadossomáticos e as observações macroscópicas, além de determinar mais precisamente a idade adulta (HernándezGallegos et al., 2002).

Outro ponto de destaque na reprodução de serpentes é que fatores ambientais, como temperatura, pluviosidade e fotoperíodo, têm influência direta, podendo levar a particularidades reprodutivas em diferentes espécies, subespécies e até mesmo espécimes inseridos em diferentes habitats, ressaltando-se a importância de se compreender essas particularidades (Girons, 1982). Dessa forma, o presente trabalho tem como objetivo caracterizar aspectos da biologia reprodutiva de Boa

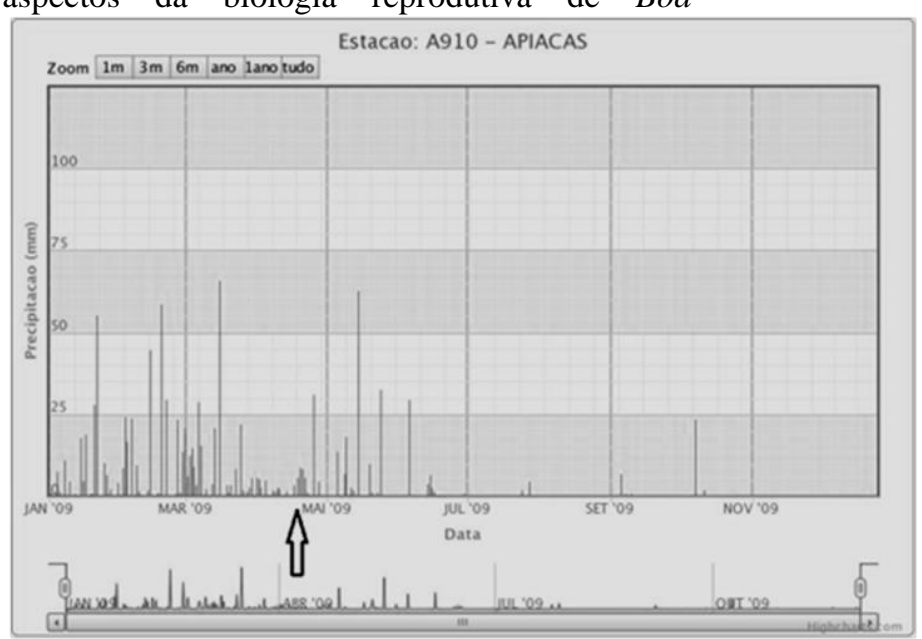

Figura 1. Índice de pluviosidade entre janeiro e dezembro de 2009, segundo a estação A910 do Instituto Nacional de Meteorologia, localizada no município de Apiacás, a cerca de $100 \mathrm{~km}$ de distância de Paranaíta, local de captura do indivíduo 7767. Pode-se observar que o período de captura (seta) caracteriza-se por índices pluviométricos elevados (Fonte: Estações automáticas - gráficos, 2018). constrictor constrictor com base nos achados histológicos dos testículos nos períodos de máxima atividade (período de gametogênese) e quiescência.

\section{MATERIAL E MÉTODOS}

Este estudo foi conduzido com autorização do Comitê de Ética no Uso de Animais (Ceua/UFMT) n ${ }^{\circ}$ 23108.204355/2017-20, para atividades com finalidade científica Sisbio/Ibama/Brasil n ${ }^{\circ}$ 57575-1. Foram obtidos os testículos de dois espécimes de Boa $c$. constrictor mantidos no Laboratório de Coleções Zoológicas - Setor de Herpetologia do Instituto de Biociências da UFMT, e nomeados 7767 e 11752. O exemplar 7767 foi capturado em 30 de abril de 2009, no município de Paranaíta, estado de Mato Grosso, Brasil. O exemplar 11752 foi capturado em 26 de setembro de 2012, no município de Castanheiras, estado de Rondônia, Brasil.

O indivíduo 7767 foi capturado no outono, período caracterizado por índices pluviométricos elevados (Fig. 1), enquanto o indivíduo 11752 foi capturado no início da primavera, período de transição entre a época seca e o início da estação chuvosa, com baixos índices de pluviosidade. (Fig. 2). Dados de precipitação dos locais de captura para o período de estudo foram obtidos do Instituto Nacional de Meteorologia, Brasil (Fonte: Estações automáticas - gráficos, 2018). 


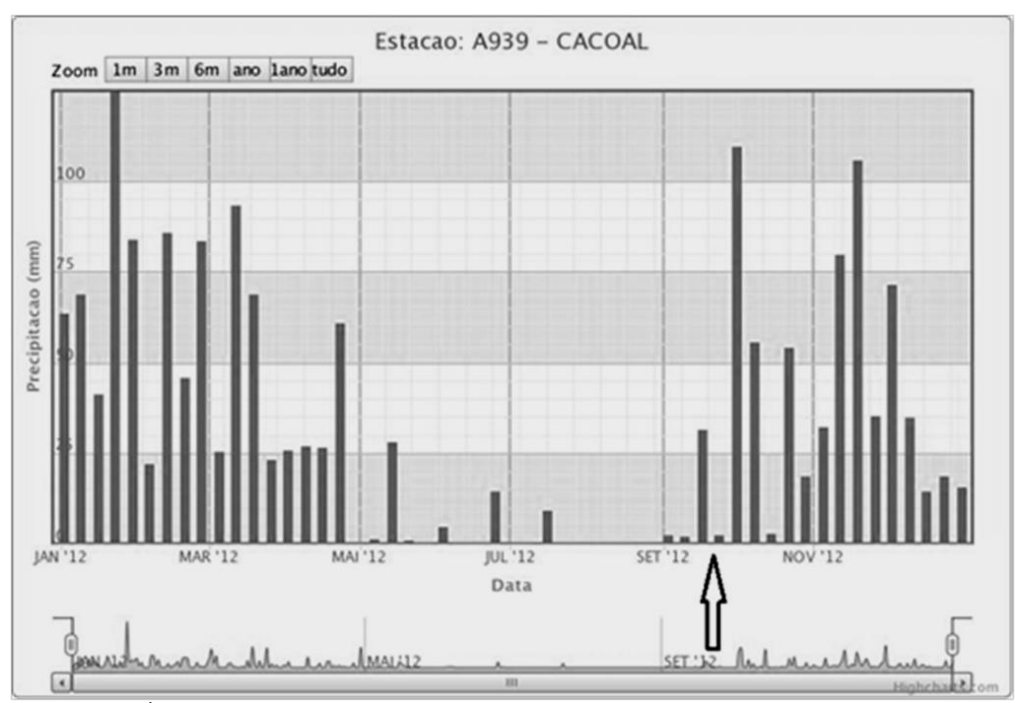

Figura 2. Índice de pluviosidade entre janeiro e dezembro de 2012, segundo a estação A939 do Instituto Nacional de Meteorologia, localizada no município de Cacoal, estado de Rondônia, a cerca de 80km de distância de Castanheiras, local de captura do indivíduo 11752. Pode-se observar que o período de captura (seta) caracteriza-se como de transição entre a época seca e o início da estação chuvosa, com baixos índices de pluviosidade (Fonte: Estações automáticas - gráficos, 2018).

Os testículos coletados foram mensurados com auxílio de fita métrica, e o volume testicular estimado calculado com base no volume de um elipsoide, segundo Méndez e Villagrán (1998). Para o processamento histológico, os testículos foram fracionados e as amostras foram desidratadas em álcool $70 \%$ e $95 \%$, respectivamente, e incorporadas em resina plástica tipo metacrilato glicol. Posteriormente, as amostras incorporadas foram cortadas a uma espessura de $3 \mu \mathrm{m}$ em micrótomo Leica RM2125 RTS e coradas com azul de toluidina $1 \%$. Por fim, foi realizada fotodocumentação, utilizandose microscópio Leica DM750, e descrição das estruturas em software Leica Application Suite, versão 3.8.0, Leica Microsystems (Switzerland) Limited.

\section{RESULTADOS}

O exemplar 7767 possuía 1,29 metro de comprimento rostro-cloacal e o exemplar 11752 possuía 1,76 metro de comprimento rostrocloacal. No primeiro indivíduo, o volume testicular era de $21,16 \mathrm{~cm}^{3}$, enquanto no segundo indivíduo o volume testicular era de $2,61 \mathrm{~cm}^{3}$. Depois de realizada a fotodocumentação e a descrição das estruturas, constatou-se que o exemplar 7767 apresentava-se em estágio de máxima atividade (período de gametogênese)
(Fig. 3), enquanto o exemplar 11752 apresentava-se em quiescência, ou seja, fora do período de gametogênese (Fig. 4).

O indivíduo que se encontrava em período de máxima atividade apresentava o lúmen dos túbulos seminíferos em evidência, com presença de espermatozoides, epitélio germinativo com células em diferentes estágios da gametogênese, além de células de Sertoli. O tecido intersticial caracterizava-se por um aparente estreitamento e os túbulos seminíferos apresentavam-se proporcionalmente maiores; células mióides com núcleo achatado foram observadas adjacentes à membrana basal, podendo-se observar no compartimento intersticial a presença de células de Leydig com tamanho proporcionalmente maior quando comparado ao indivíduo em período quiescente. Nos compartimentos basais dos túbulos seminíferos, observou-se a presença de espermatogônias, sendo essas células arredondadas com núcleo evidente; dois estágios de espermatogônias foram observados: células com núcleo arredondado, escuro e citoplasma pouco evidente (espermatogônias escuras ou de tipo A), e células com núcleo arredondado, menor e citoplasma evidente (espermatogônias claras ou de tipo B). Ainda no epitélio germinativo, observou-se a presença de espermatócitos primários e secundários, este 
último dando origem a espermátides, as quais apresentavam, em sua última fase, cauda alongada projetando-se para o lúmen dos túbulos seminíferos e, por fim, diferenciando-se em espermatozoides.
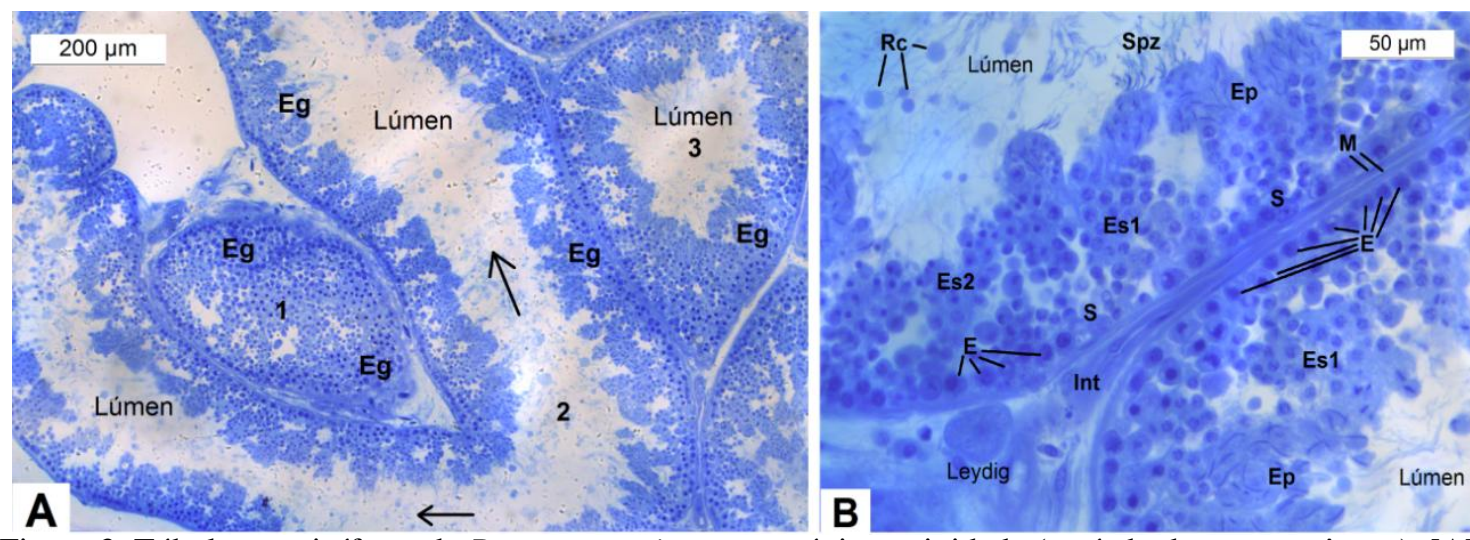

Figura 3. Túbulos seminíferos de Boa c. constrictor em máxima atividade (período de gametogênese). [A] Túbulos seminíferos em corte sagital (1), longitudinal (2) e transversal (3); as setas indicam a extensão do túbulo seminífero em um corte longitudinal. É possível observar um aumento na dimensão dos túbulos seminíferos e consequente estreitamento do tecido intersticial. Legenda: epitélio germinativo (Eg). Azul de toluidina 1\%, obj. 10x. [B] É possível visualizar células em diferentes fases da gametogênese e presença de espermatozoides na luz do túbulo seminífero; célula de Leydig evidente no interstício. Legenda: espermatogônia (E), espermatócito primário (Es1), espermatócito secundário (Es2), espermátide (Ep), espermatozoide (Spz), resquício citoplasmático (Rc), célula de Sertoli (S), célula mioide (M), tecido intersticial (Int). Azul de toluidina 1\%, obj. 40x.
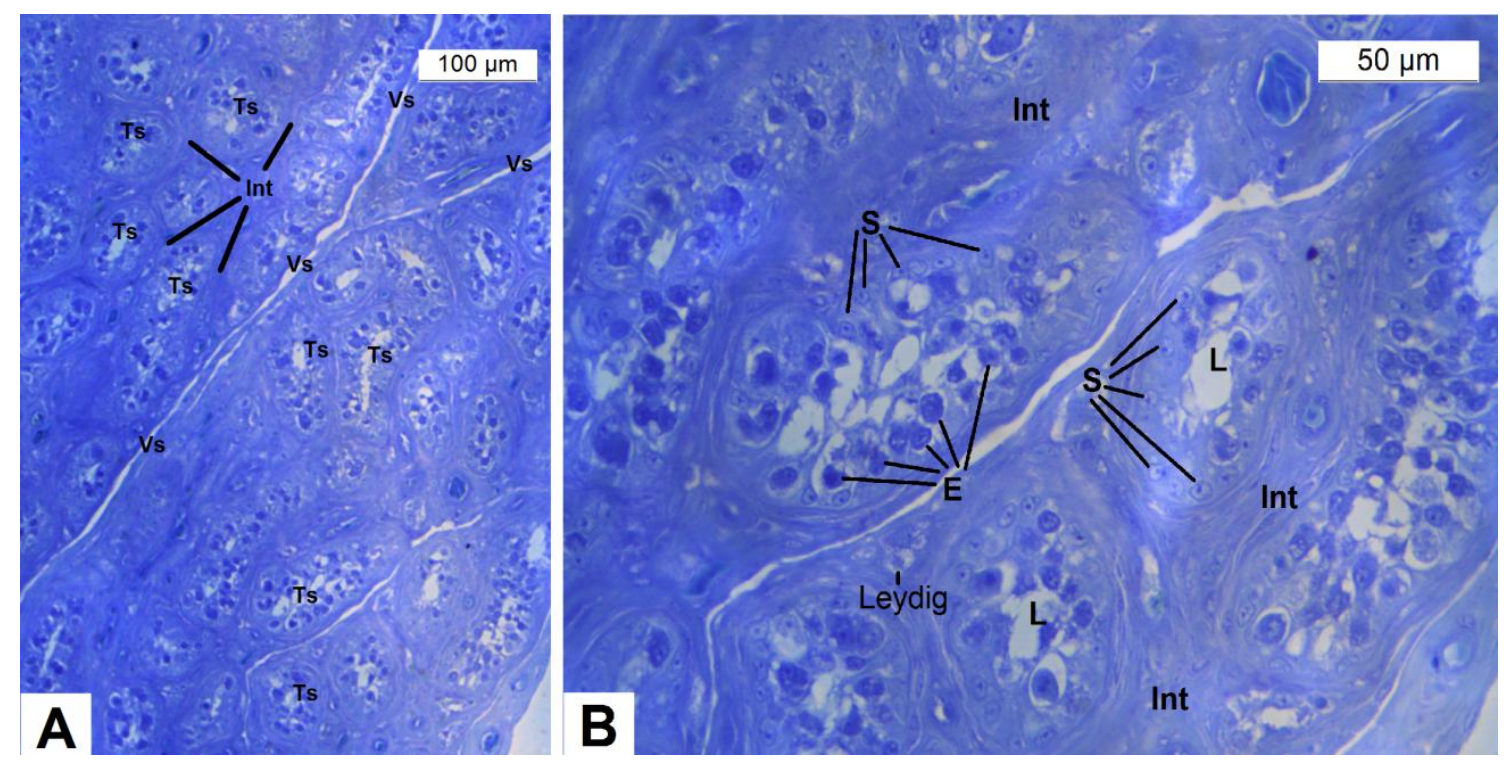

Figura 4. Túbulos seminíferos de Boa c. constrictor em período quiescente. [A] É possível observar uma diminuição na dimensão dos túbulos seminíferos e consequente aumento do tecido intersticial; vasos sanguíneos tornam-se evidentes. Legenda: túbulo seminífero (Ts), vaso sanguíneo (Vs). Azul de toluidina 1\%, obj. 10x. [B] Presença de células de Sertoli e espermatogônias no epitélio germinativo no período quiescente; nota-se o estreitamento da luz dos túbulos seminíferos. Legenda: espermatogônia (E), célula de Sertoli (S), lúmen (L), tecido intersticial (Int). Azul de toluidina 1\%, obj. 40x. 
O indivíduo que se encontrava em período quiescente apresentava o lúmen dos túbulos seminíferos pouco evidentes, e tecido intersticial espesso, sendo a relação tecido intersticial/túbulo seminífero o inverso do observado no indivíduo em máxima atividade. As células de Leydig presentes no indivíduo quiescente apresentavamse menores quando comparadas às do indivíduo em período de gametogênese, e os vasos sanguíneos tornaram-se evidenciados. No compartimento germinativo, foi possível observar apenas espermatogônias escuras (ou do tipo A) e células de Sertoli.

\section{DISCUSSÃO}

Em trabalho realizado com Sceloporus jarrovi, os estágios testiculares são expressos em oito fases: (0) juvenil: células germinativas não aparentes; (1) crescente: divisão de células germinativas sem o desenvolvimento do lúmen; (2) espermatogênese precoce: espermatócitos primários na margem luminal; (3) espermatogênese: espermatócitos secundários na margem luminal; (4) espermiogênese: espermátides indiferenciadas na margem luminal; (5) maduros: espermatozoides em metamorfose na margem luminal; (6) reprodutivos: espermatozoides maduros na luz; (7) pós-reprodutivos: regressão precoce e presença de restos celulares no lúmen; (8) inativos: regressão completa, sem divisão celular e sem lúmen (Ballinger e Nietfeldt, 1989).

A presença de espermatozoides na luz do túbulo seminífero no indivíduo 7767 indica um período de máxima gametogênese, enquanto o lúmen dos túbulos seminíferos pouco evidentes, sem a presença de espermatozoides e de células gaméticas em divisão, caracteriza o indivíduo 11752 em período quiescente. Presença de espermatócitos, espermátides e espermatozoides na luz dos túbulos seminíferos também é observada em machos de Gloydius halys caucasicus e Boa c. occidentalis em período de máxima gametogênese (Ibargüengoytía et al., 2006; Salehi et al., 2018). Células de Sertoli são observadas em ambos os períodos reprodutivos, porém estudo realizado com serpentes que apresentam gametogênese sazonal mostra que essas células desempenham funções apenas em período de máxima atividade gamética (Salehi et al., 2018).
No presente trabalho, evidencia-se uma correlação inversa entre as células de Leydig, ou seja, estas se tornam maiores e mais evidentes no indivíduo em gametogênese quando comparado ao indivíduo em período quiescente. Variações morfológicas das células de Leydig são observadas em outros répteis; em Gloydius halys caucasicus, observa-se atividade das células de Leydig em todos os períodos reprodutivos, no entanto estas se tornam maiores nos períodos de cópula (Salehi et al., 2018). Em Iguana iguana, que apresenta gametogênese sazonal, variações morfológicas nas células de Leydig também são relatadas (Ferreira et al., 2002).

Estudos realizados por meio de avaliação histológica (Ibargüengoytía et al., 2006) e ultrossonográfica (Bertona e Chiaraviglio, 2003) dos testículos de Boa c. occidentalis constataram que indivíduos classificados como adultos, em reprodução, apresentam testículos túrgidos, com o período de máxima gametogênese ocorrendo ao final da estação chuvosa, enquanto no período seco os machos apresentam testículos quiescentes, fora do período de gametogênese. $\mathrm{O}$ mesmo fato foi observado no presente estudo, visto que o período de captura do indivíduo em gametogênese caracteriza-se como período das águas (Fig. 1), com considerável índice pluviométrico, enquanto o indivíduo quiescente foi capturado em um período caracterizado pelos baixos índices de pluviosidade, ao final do período seco (Fig. 2).

No indivíduo em período de máxima atividade, evidencia-se um volume maior do testículo quando comparado ao indivíduo quiescente. Esses achados corroboram a avaliação ultrassonográfica descrita por Garcia et al. (2015), em que testículos de Eunectes murinus, Boa c. constrictor e Epicrates cenchria apresentavam-se maiores no período reprodutivo, e vão de encontro ao observado em descrição histológica dos testículos de Boa c. occidentalis (Bertona e Chiaraviglio, 2003; Ibargüengoytía et al., 2006), Sceloporus palaciosi (Méndez e Villagrán, 1998) e Gloydius halys caucasicus (Salehi et al., 2018). O volume testicular é um resultado não apenas da espermatogênese, mas também da atividade endócrina, e, por isso, correlacionar apenas os estágios espermáticos com tamanho testicular pode ser um engano. Crotalus scutulatus, por exemplo, apresenta um volume testicular máximo no período de 
regressão como consequência da atividade endócrina (Schuett et al., 2002; Ibargüengoytía et al., 2006).

Serpentes podem apresentar sazonalidade quanto à produção espermática. Em machos, ciclos sazonais em que a produção de células gaméticas ocorra no mesmo período ou no período que precede a época de cópula são denominados prénupciais ou associados, enquanto naqueles indivíduos em que a produção gamética ocorre após o período de cópula e consequentemente há estocagem de esperma nos ductos deferentes até que esta ocorra são denominados pós-nupciais ou dissociados (Girons, 1982). Bertona e Chiaraviglio (2003) descrevem que o período de sincronização e cópula em Boa c. occidentalis ocorre no período seco e que essa característica, associada com o período de máxima gametogênese do macho no período chuvoso, sugere um padrão pré-nupcial.

Em Boa c. constrictor, o período de cópula ocorre no outono-inverno (Garcia, 2012), período caracterizado pelos índices pluviométricos mais baixos. O padrão de sazonalidade associado à gametogênese observado no presente estudo, juntamente com o período de cópulas relatado, é semelhante ao observado em Boa c. occidentalis, pressupondo que o padrão reprodutivo de Boa. c. constrictor seja pré-nupcial.

\section{CONCLUSÃO}

Os achados histológicos descritos no presente estudo constatam que Boa constrictor constrictor apresenta sazonalidade em relação à gametogênese, com massa testicular maior nos indivíduos em máxima atividade. A gametogênese ocorre nos períodos com alto índice pluviométrico, enquanto no período caracterizado pelos baixos índices de pluviosidade os indivíduos encontram-se quiescentes. Esse padrão de sazonalidade associado ao período de cópulas relatado em literatura caracteriza Boa c. constrictor como serpente com padrão pré-nupcial.

\section{AGRADECIMENTOS}

Os autores agradecem à Coordenação de Aperfeiçoamento de Pessoal de Nível Superior (Capes), pela bolsa concedida a Heitor José
Bento; ao Laboratório de Coleções Zoológicas Setor de Herpetologia do Instituto de Biociências da UFMT, por disponibilizar os exemplares utilizados neste estudo; e ao Laboratório de Análises Morfológicas e Morfométricas do Instituto de Biociências da UFMT, por disponibilizar a estrutura e o apoio intelectual para o desenvolvimento desta pesquisa.

\section{REFERÊNCIAS}

BALLINGER, R.E.; NIETFELDT, J.W. Ontogenetic stages of reproductive maturity in the viviparous tizard, Sceloporus jarrovi (Iguanidae). J. Herpetol., v.23, p.282-292, 1989.

BÉRNILS, R.S.; COSTA, H.C. Brazilian reptiles: list of species. Version 2012.1. Sociedade Brasileira de Herpetologia, 2012. Disponível em: <http://www.sbherpetologia.org.br/>. Acessado em: 14 abr. 2018.

BERTONA, M.; CHIARAVIGLIO, M. Reproductive biology, mating aggregations, and sexual dimorphism of the Argentine boa constrictor (Boa constrictor occidentalis). J. Herpetol., v.37, p.510-516, 2003.

ESTAÇÕES automáticas - gráficos. Brasília: INMET, 2018. Disponível em: <http://www.inmet.gov.br/portal/index.php?r=ho $\mathrm{me} /$ page\&page $=$ rede_estacoes_auto_graf $>$.

Acessado em: 14 jun. 2018.

FERREIRA, A.; LAURA, I.A.; DOLDER, H. Reproductive cycle of male green iguanas, Iguana iguana (Reptilia: Sauria: Iguanidae), in the Pantanal region of Brazil. Braz. J. Morphol. Sci., v.19, p.23-28, 2002.

GARCIA, V.C.; VAC, M.H.; BADIGLIAN, L.; ALMEIDA-SANTOS, S.M. Avaliação ultrassonográfica do aparelho reprodutor em serpentes vivíparas da família Boidae. Pesqui. Vet. Bras., v.35, p.311-318, 2015.

GARCIA, V.C. Avaliações ultrassonográficas dos ciclos reprodutivos das serpentes Boidae Neotropicais. 2012. 101f. Dissertação (Mestrado em Anatomia dos Animais Domésticos e Silvestres) - Universidade de São Paulo, São Paulo, SP. 
GIRONS, H.S. Reproductive cycles of male snakes and their relationships with climate and female reproductive cycles. Herpetologica, v.38, p.5-16, 1982.

GREGO, K.F.; ALBUQUERQUE, L.R.; KOLESNIKOVAS, C.K.M. Squamata (Serpentes). In: CUBAS, Z.S.; SILVA, J.C.R.; CATÃO-DIAS, J.L. (Eds.). Tratado de animais selvagens: medicina veterinária. 2.ed. São Paulo: Roca, 2014. p.186-218.

HERNÁNDEZ-GALLEGOS, O.; CRUZ, F.R.M.; CRUZ, M.V.; ANDREWS R.M. Continuous spermatogenesis in the lizard Sceloporus bicanthalis (sauria: phrynosomatidae) from high elevation habitat of central Mexico. Herpetologica, v.58, p.415-421, 2002.

IBARGÜENGOYTÍA, N.R.; BERTONA, M.; CHIARAVIGLIO, M. Seasonal changes in testicular activity of the protected cites i Boa constrictor occidentalis (Serpentes: Boidae): an histological study. S. Am. J. Herpetol., v.1, p.143-148, 2006.

MÉNDEZ, F.R.; VILLAGRÁN, M. Reproducción asincrónica de Sceloporus palaciosi (Sauria: Phrynosomatidae) en México, con comentarios sobre sus ventajas y regulación. Rev. Biol. Trop., v.46, p.1159-1161, 1998.
PIZZATTO, L.; ALMEIDA-SANTOS, S.M.; MARQUES. O.A.V. Biologia reprodutiva de serpentes brasileiras. In: OLIVEIRA, M.E.; BARRETO, L. (Eds.). Herpetologia no Brasil. Piraquara: Sociedade Brasileira de Herpetologia, 2006. v.2, p. 201-221.

SALEHI, A.; TODEHDEHGHAN, F.; ESLIMIESFAHANI, D. Testosterone level, testicular histology and reproductive events of caucasian pit viper, Gloydius halys caucasicus (Serpentes: Viperidae). J. Adv. Biol., v.11, p.2155-2162, 2018.

SCHUETT, G.W.; CARLISLE, S.L.; HOLYCROSS, A.T. et al. Mating system of male mojave rattlesnakes (Crotalus scutulatus): seasonal timing of mating, agonistic behavior, spermatogenesis, sexual segment of the kidney, and plasma sex steroids. In: SCHUETT, G.W.; HOGGREN, M.; DOUGLAS, M.; GREENE, H. (Eds.). Biology of vipers. Eagle Mountain: Eagle Mountain Publishing, 2002. p.515-532.

UETZ, P.; FREED, P.; HOŠEK, J. The reptile database. 2018. Available in: <http://www.reptile-database.org>. Accessed in: 18 Jun. 2018.

ZUG, G.R.; VITT, L.J.; CALDWELL, J.P. Snakes, In:. (Eds). Herpetology. 2.ed. San Diego: Academic Press, 2001. p.503-595. 